\title{
SIMULATION STUDY OF THE OPTIMAL APPOINTMENT NUMBER FOR OUTPATIENT CLINICS
}

\author{
Zhu, Z. C.; Heng, B. H. \& Teow, K. L. \\ Department of Health Services \& Outcomes Research, National Healthcare Group, Singapore \\ 6 Commonwealth Lane, Level 04-01, GMTI Building, Singapore 149547 \\ E-Mail: zhecheng_zhu@nhg.com.sg
}

\begin{abstract}
This paper analyzes the appointment scheduling systems in specialist outpatient clinics (SOC) to determine the optimal number of appointments to be planned in one session under different performance indicators and consult room configurations. A discrete event simulation model is constructed to model the workflow of a SOC considering three consult room configurations. Ground data are collected to generate model parameters including consult time, no-show rate and lateness rate. An appointment rule based on Bailey's rule is proposed to generate appointments with various numbers of planned appointments in one session. Simulation results are measured by performance indicators including patient queue, patient waiting time, doctor utilization and doctor overtime. Simulation results show that the optimal number of planned appointments varies according to different operational requirements and consult room configurations. Study in this paper provides a guideline to choose the optimal number of planned appointments according to specific requirements.

(Received in December 2008, accepted in May 2009. This paper was with the authors 2 months for 2 revisions.)
\end{abstract}

Key Words: Appointment Scheduling, Discrete Event Simulation, Outpatient Clinics

\section{INTRODUCTION}

An outpatient clinic is a private or public healthcare facility which is devoted to diagnoses and treatments of outpatients [1]. There are many types of outpatient clinics and functions of clinics vary from country to country [2]. For example, a general outpatient clinic provides general treatments for patients of all kinds of diseases. A specialist clinic offers sophisticated diagnoses and treatments for a specific disease. In Singapore, there are mainly three types of outpatient clinics: general practitioner (GP), polyclinic and specialist outpatient clinics (SOC). GP is a private medical practitioner who provides consultation and medicine in the neighbourhood. Polyclinic is a government clinic covering a wide range of treatments for subsidized patients. SOC is clinics associated with hospitals and medical centres. Each SOC is specialized on one type of diseases, e.g., orthopedic clinic, ear, nose and throat clinic, eye clinic.

Among the three types of outpatient clinics, GP and polyclinic mainly accept walk-in patients and SOC mainly accepts patients with appointments. Patients are referred to SOC by GP or polyclinics. Schedulers in SOC make an appointment for each referred patient by picking up a free slot of a specific specialist through the appointment management system. The number of available slots is determined by multiple factors, e.g., doctor workload, target revenue. Compared to walk-in based clinic, SOC has several advantages [3], e.g., patients are able to see their preferred doctors and continue to see the same doctor for their follow-up visits; waiting time of patients with appointments is usually less than those walk-in patients.

An SOC itself is a complex system which contains complex workflow and various uncertainties. Many constraints should be considered during the appointment scheduling phase. One the system side, many doctors work in an SOC simultaneously. Hence the 
scheduling system is required to handle multiple appointment lists and solve possible conflicts on resources. On the patient side, each patient has his preferred doctor. Patient flow varies case by case, e.g., pre or post lab tests or x-ray check incur to some patients. On the doctor side, each doctor has his preference on his appointment such as the number of patients arranged in one session. Besides above mentioned constraints, numbers of uncertainties exist in an SOC. For example, patients may not show up on the day of their appointments and may arrive later than the scheduled appointment time. Further, the consult time of each patient varies case by case. All above mentioned uncertainties cause fluctuation and are possible factors leading to long patient waiting time and doctor overtime in an SOC.

In this paper, study is conducted to improve the appointment scheduling of an orthopedic clinic (Clinic B) in a public hospital in Singapore. Clinic B is a big SOC including 18 consult rooms and 50 doctors. Three types of consult room configurations are currently applied in Clinics B, which are respectively consult rooms with one doctor, two doctors and three doctors. Four key performance indicators are used to measure the performance of Clinic B, which are respectively patient queue, patient waiting time, doctor utilization and doctor overtime. Currently, problems existing in Clinic B include long patient queue, long patient waiting patient and doctor overtime. The management wishes to apply better appointment schedules to improve SOC performance measured by four key performance indicators.

Appointment rules are methods used to generate an appointment schedule by arranging the order and time slots for each appointment. Numbers of appointment rules have been proposed to resolve the problem of long patient waiting time and doctor overtime. As early as the 1950s, [4] proposed an appointment rule known as the Bailey's rule. The basis of Bailey's rule is to book two appointments at the beginning of a session, which is known as initial block. Successive appointments are then booked one by one at the interval of mean consult time. A similar rule was proposed in [5, 6]. Other than booking one patient in one time slot, several research works also proposed the idea of booking multiple patients in one time slot. [7] proposed a rule which booked two patients in each time slot. [8, 9] argued that it was better to book different number of patients in different time slots.

All the appointment rules mentioned above have fixed intervals between two successive appointments. There are quite a few rules with flexible intervals, which can be classified into two approaches. The first approach determines the intervals by patient type. Such an approach is quite popular in real application. It is a common practice for clinic staff to assign different intervals for new patients and follow-up patients [10]. The second approach adjusted the intervals according to different time of the session. [11] pointed out that it was helpful to arrange a longer interval at the latter part of the session compared to the early part of the session. [12] pointed out that the intervals should follow an increase then decrease manner. For a more comprehensive review of various appointment rules, refer to [13, 14].

In this paper, an appointment rule based on Bailey's rule is proposed to help Clinic B reduce patient waiting time and doctor overtime. Compared to the original Bailey's rule, the new proposed appointment rule handles various consult room configurations by assigning different size of initial blocks. The appointment interval is also adjustable according to the demand. The newly proposed appointment rule is more appropriate in applications described in this paper because there are three consult room configurations in Clinic B and the demand for each consult room varies greatly.

Discrete event models are widely applied to simulate the appointment scheduling problems in outpatient system [15-19]. In this paper, a discrete event model is constructed to simulate the workflow of Clinic B. Three consult room configurations are modelled respectively. Various appointment schedules generated by the newly proposed appointment rule are tested in the simulation model. Relationship between the number of planned appointments and four key performance indicators on different confidence levels is provided. 
The rest of this paper is organized as follows: Workflow of SOC is described followed by a discrete simulation model based on the workflow; Simulation results and analysis are then given; conclusion is drawn in the end.

\section{METHODS AND MODELS}

\subsection{Workflow of SOC}

Workflow of the SOC studied in this paper is illustrated in Fig. 1. A patient arrives at the SOC and registers at the registration counter first. He then joins the queue outside the consult room where his appointed specialist is stationed. When his name is called, he enters the consult room to begin his consultation. When the consultation is over, he proceeds to the cash counter to pay the bill and leaves the SOC. Patient no-show and lateness is considered in this paper. Some patients in the appointment schedule may not show up on the day of their appointments and some patients may come later than the appointment time.

Three different consult room configurations are considered in this paper:

1. Consult room with one doctor. One doctor in the consult room. All patients visit the doctor once.

2. Consult room with two doctors. One senior doctor and one junior doctor work together in one consult room. The junior doctor conducts the first consultation supervised by the senior doctor. The senior doctor conducts the second consultation if needed. All patients go through the first consultation, $20 \%$ of the patients go through the second consultation.

3. Consult room with three doctors. Three doctors cooperate in one consult room. Each doctor is in charge of one of three procedures including wound inspection, consultation and cast. One patient goes though one or more procedures in sequence. $35 \%$ of the patients go through wound inspection, $70 \%$ of the patients go through consultation and 20 $\%$ of the patients go through cast.

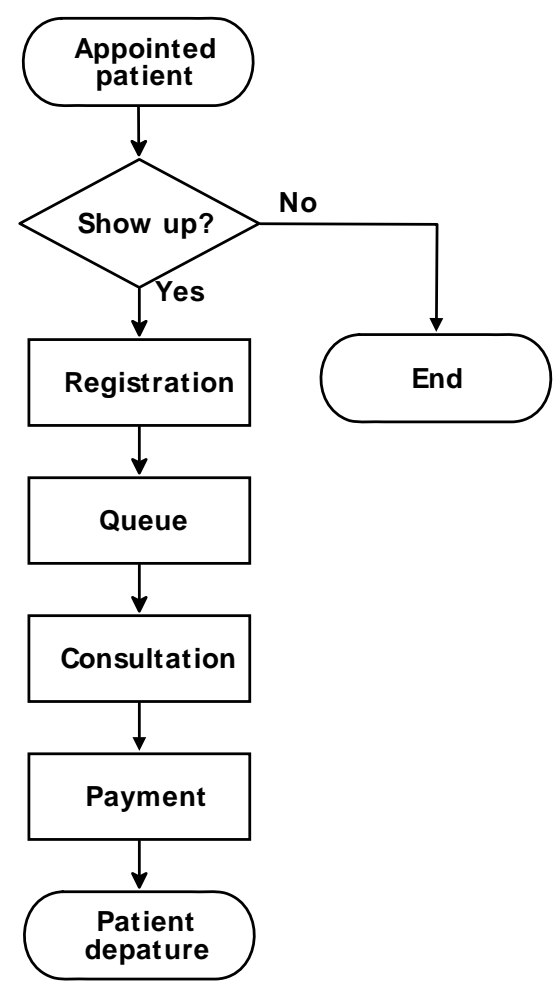

Figure 1: Workflow of SOC studied in this paper. 


\subsection{Construction of simulation model}

Fig. 2 illustrates the simulation model based on the workflow described in the previous section. Several assumptions are made based on the ground data collected:

- Registration time and payment time is negligible compared to the consult time. Hence they are not considered in this paper.

- Each patient visits one of three consult rooms once and only once.

- No significant difference of consult time between new patients and follow-up patients. Hence such difference is not considered in this paper.

- All doctors are punctual and the difference of consult time among doctors is not considered.

- Patient earliness is not considered in this paper.

- The SOC is purely appointment-based. No walk-in patients are considered.

The simulation model was constructed using Simul8 ${ }^{\circledR} 2008$ professional [20]. Internal mechanisms such as patient arrival, no-show, lateness and routes inside the clinic were constructed by Visual Logic $^{\circledR}$ [21], a scripting language provided in Simul8 ${ }^{\circledR} 2008$ professional. Parameters in the simulation models are listed as follows:

- Session duration. The opening hours of a specific SOC.

- Distribution of arrival. The interval between two consecutive arrivals.

- No-show rate. Ratio of the patients who do not show up on the day of their appointments.

- Lateness rate. Ratio of the patients who arrive at the SOC later than their appointment time.

- Distribution of consult time. Time spent in the consult room for each patient.

Manual survey was conducted in Clinic B to collect ground data for a period of two weeks. A survey form was used to keep track of the activity of each patient including arrival time, start time, leaving time, etc, which were used to estimate model parameters. Estimated parameters are listed in Table I.

Discrete event simulation of an SOC

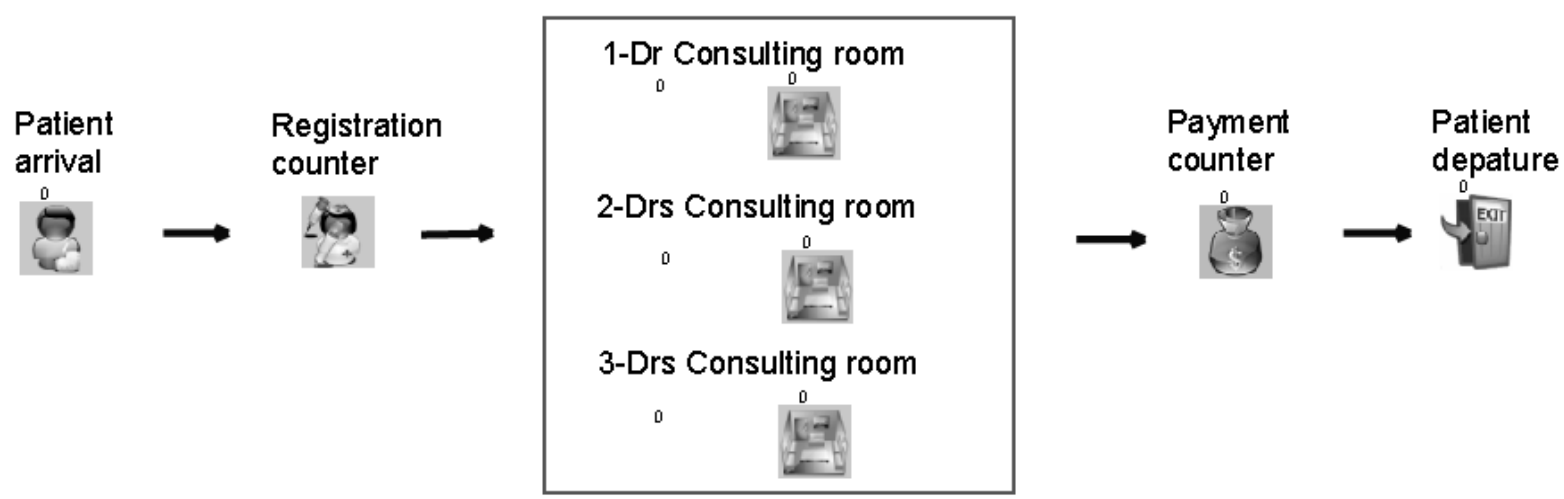

Figure 2: Simulation model of an SOC with three types of consult room configurations.

\subsection{Rule to generate appointment schedule}

The appointment rule applied in this paper extends Bailey's rule in the following two ways: Firstly, two-doctor and three-doctor situations are considered. Secondly, the interval between two appointments is determined by the number of planned appointments per session. 
Table I: Parameters of the simulation model.

\begin{tabular}{|c|c|}
\hline Session duration & $\begin{array}{l}\text { AM session: 9:00-12:30 } \\
\text { PM session: 14:00-17:30 }\end{array}$ \\
\hline Distribution of arrival & $\begin{array}{l}t+\Delta \\
t \text { denotes the appointment time } \\
\Delta \text { denotes patient lateness }\end{array}$ \\
\hline No-show rate & $20 \%$ \\
\hline Lateness rate & $11.5 \%$ \\
\hline Distribution of lateness $(\Delta)$ & Gamma $(9.4,1.6)^{*}$ \\
\hline Distribution of consult time & $\begin{array}{l}\text { One-doctor consult room: Gamma }(5.8,1.2) \\
\text { Two-doctor consult room: Gamma }(4.0,2.5) \text { for junior doctor, } \\
\text { Lognormal }(1.2,0.9)^{* *} \text { for senior doctor. } \\
\text { Three-doctor consult room: Lognormal }(2.2,0.5) \text { for wound } \\
\text { inspection, Gamma }(3.3,3.2) \text { for consultation, Lognormal }(2.3 \text {, } \\
0.2) \text { for cast. }\end{array}$ \\
\hline
\end{tabular}

The rule is described as follows:

- The number of appointments $d$ at the beginning of a session is determined by (1):

$$
d= \begin{cases}2, & \text { one - doctor consulting room } \\ 3, & \text { two - doctor consulting room } \\ 5, & \text { three - doctor consulting room }\end{cases}
$$

- The interval $I$ between two appointments is calculated by (2):

$$
I=\frac{T-C}{N-d}
$$

where $N$ denotes the number of planned appointments. $T$ denotes the session duration. $C$ denotes the cleanup period, a buffer period for a doctor to handle all incomplete appointments before the end of session.

All appointments are scheduled before the cleanup period. For example, considering a two-doctor consult room, the session duration is 210 minutes, the cleanup period is 30 minutes, the number of planned appointments is 30 . All 30 appointments should be scheduled 30 minutes before the end of session. 3 appointments are scheduled at the start of session. The interval between the follow-up appointments is calculated by (2), which is 6.67 minutes.

\section{RESULTS AND ANALYSIS}

All simulations were conducted on a PC with an AMD Athlon X2 1.8GHz CPU. The operating system was Windows XP SP3. The number of planned appointments ranged from 15 to 60 to reflect the congestion of a consult room. 20 instances were simulated for each number of planned appointments. Four key performance indicators were used to measure the performance of the consult rooms. They are respectively patient waiting time, patient queue, doctor overtime and doctor utilization. Patient waiting time in this paper is defined as consult start time - max(appointment time, arrival time). Relationship between the number of planned appointments and four key performance indicators is given on three confidence levels: $50^{\text {th }}$ percentile (median level), $75^{\text {th }}$ percentile (majority level) and $95^{\text {th }}$ percentile (extreme level). 


\subsection{Results and analysis of consult room with one doctor}

Table II illustrates the result summary of the consult room with one doctor. The number of planned appointments in one session ranges from 15 to 50. Fig. 3 illustrates the relationship between four key performance indicators and the number of planned appointments per session.

The $50^{\text {th }}$ percentile results show the median cases. It is observed from Fig. 3(a) and Fig. 3(b) that when the number of planned appointments is less than 25 in one session, no queue forms outside the consult room and the patient waiting time is 0. It is observed from Fig. 3(c) and Fig. 3(d) that in median case, doctors do not work overtime when the number of planned appointments is 35 or less.

The $95^{\text {th }}$ percentile results show the worst cases. It is observed from Fig. 3(a) and Fig. 3(b) that queue forms even when the number of planned appointments is 15 and the corresponding waiting time is about 4 minutes. It is observed from Fig. 3(c) and Fig. 3(d) that in worst case, doctors have to work overtime when the number of planned appointments is 35 or more.

Table II: Result summary of consult room with one doctor.

\begin{tabular}{|c|c|c|c|c|c|c|c|c|c|c|c|c|}
\hline \multirow[b]{2}{*}{$\mathrm{N}$} & \multicolumn{3}{|c|}{ Queue } & \multicolumn{3}{|c|}{ Waiting time (Mins) } & \multicolumn{3}{|c|}{ Overtime (Mins) } & \multicolumn{3}{|c|}{ Utilization (\%) } \\
\hline & $50 \%$ & $75 \%$ & $95 \%$ & $50 \%$ & $75 \%$ & $95 \%$ & $50 \%$ & $75 \%$ & $95 \%$ & $50 \%$ & $75 \%$ & $95 \%$ \\
\hline 15 & 0 & 0 & 1 & 0 & 0 & 4.1 & 0 & 0 & 0 & 38 & 40 & 47 \\
\hline 20 & 0 & 1 & 1 & 0 & 0.2 & 6.5 & 0 & 0 & 0 & 51 & 53 & 62 \\
\hline 25 & 0 & 1 & 2 & 0 & 3.6 & 7.8 & 0 & 0 & 0 & 65 & 67 & 70 \\
\hline 30 & 1 & 2 & 3 & 4.2 & 8.5 & 15.9 & 0 & 0 & 0 & 80 & 81 & 87 \\
\hline 35 & 2 & 3 & 4 & 10.7 & 15.5 & 27.0 & 0 & 0 & 9.8 & 91 & 97 & 103 \\
\hline 40 & 2 & 4 & 6 & 12.7 & 25.4 & 41.2 & 10.5 & 16.2 & 24.9 & 100 & 106 & 111 \\
\hline 45 & 5 & 7 & 11 & 29.9 & 50.7 & 74.5 & 39.5 & 56.0 & 68.5 & 118 & 125 & 132 \\
\hline 50 & 8 & 11 & 16 & 49.3 & 72.2 & 102.7 & 70.5 & 82.7 & 100.9 & 132 & 139 & 147 \\
\hline
\end{tabular}

(a) Patient queue vs $\mathrm{N}$

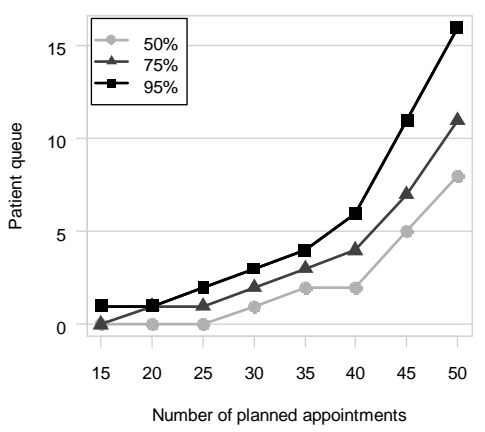

(c) Doctor overtime vs $\mathrm{N}$

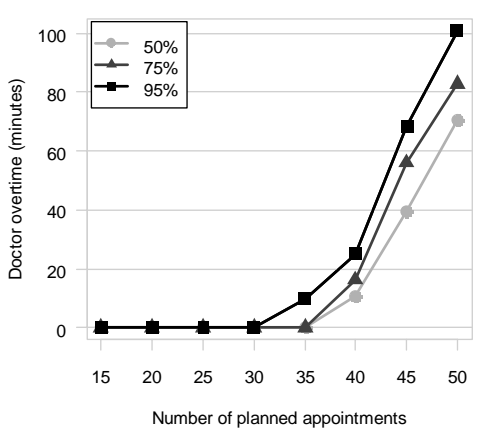

(b) Patient waiting time vs $\mathrm{N}$

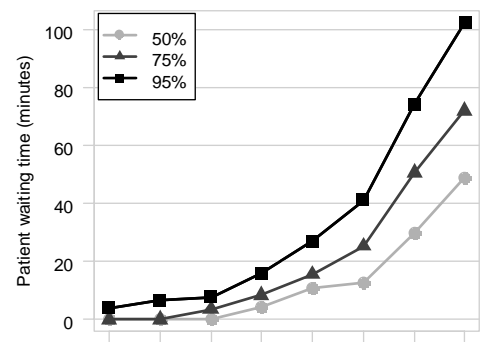

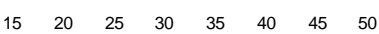

Number of planned appointments

(d) Doctor utilization vs $\mathrm{N}$

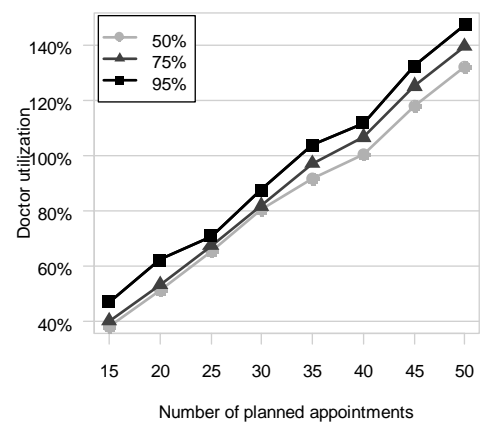

Figure 3: Relationship between performance indicators and number of planned appointments per session in one-doctor consult room. 


\subsection{Results of consult room with two doctors}

Table III illustrates the results summary of the consult room with two doctors. The number of appointment in one session ranges from 25 to 60. Fig. 4 illustrates the relationship between four key performance indicators and the number of planned appointments per session.

It is observed from Fig. 4(a) and Fig. 4(b) that in the median case (50\%), when the number of planned appointments is 30 or less, no queue forms outside the consult room and the patient waiting time is 0. It is observed from Fig. 4(c) and Fig. 4(d) that in median case (50\%), doctors do not work overtime when the number of planned appointments is 35 or less.

It is observed from Fig. 4(a) and Fig. 4(b) that in worst case (95\%), queue forms when the number of planned appointments is 25 and the corresponding waiting time is about 6 minutes. It is observed from Fig. 4(c) and Fig. 4(d) that in worst case (95\%), doctors have to work overtime when the number of planned appointments is 40 or more.

Table III: Result summary of consult room with two doctors.

\begin{tabular}{|r|r|r|r|r|r|r|r|r|r|r|r|r|}
\hline \multirow{2}{*}{$\mathrm{N}$} & \multicolumn{4}{|c|}{ Queue } & \multicolumn{3}{|c|}{ Waiting time (Mins) } & \multicolumn{3}{c|}{ Overtime (Mins) } & \multicolumn{3}{|c|}{ Utilization (\%) } \\
\cline { 2 - 16 } & 50 & $75 \%$ & $95 \%$ & $50 \%$ & $75 \%$ & $95 \%$ & $50 \%$ & $75 \%$ & $95 \%$ & $50 \%$ & $75 \%$ & $95 \%$ \\
\hline 25 & 0 & 0 & 1 & 0 & 0 & 5.9 & 0 & 0 & 0 & 59 & 60 & 66 \\
\hline 30 & 0 & 1 & 2 & 0 & 4.8 & 13.4 & 0 & 0 & 0 & 76 & 81 & 83 \\
\hline 35 & 1 & 2 & 3 & 5.5 & 9.6 & 16.3 & 0 & 0 & 0 & 87 & 93 & 95 \\
\hline 40 & 1 & 4 & 7 & 13.4 & 24.3 & 40.1 & 4.5 & 14.5 & 32.3 & 97 & 104 & 113 \\
\hline 45 & 3 & 6 & 10 & 22.0 & 36.7 & 60.5 & 30.5 & 40.7 & 57.5 & 112 & 117 & 126 \\
\hline 50 & 4 & 8 & 12 & 34.3 & 52.3 & 73.1 & 46.0 & 55.7 & 71.0 & 119 & 125 & 131 \\
\hline 55 & 6 & 10 & 15 & 43.9 & 63.4 & 98.9 & 78.5 & 86.0 & 100.1 & 133 & 138 & 146 \\
\hline 60 & 10 & 15 & 21 & 62.2 & 97.3 & 131.8 & 101.0 & 126.0 & 149.9 & 146 & 159 & 169 \\
\hline
\end{tabular}

(a) Patient queue vs $\mathrm{N}$

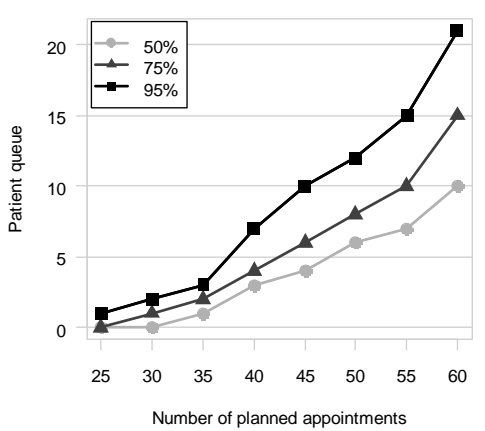

(c) Doctor overtime vs $\mathrm{N}$

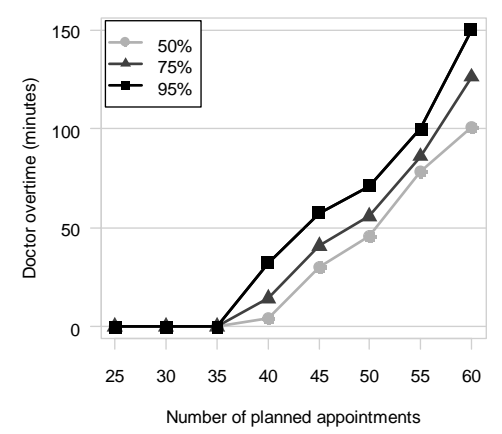

(b) Patient waiting time vs $\mathrm{N}$

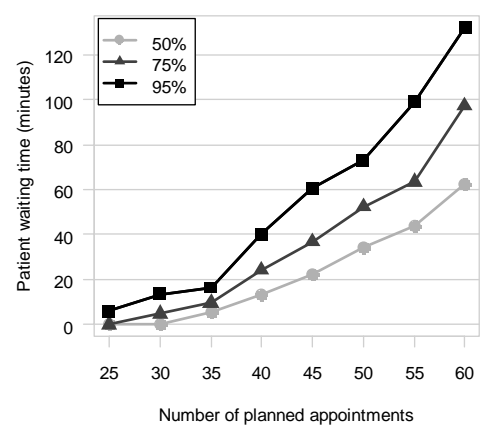

(d) Doctor utilization vs $\mathrm{N}$

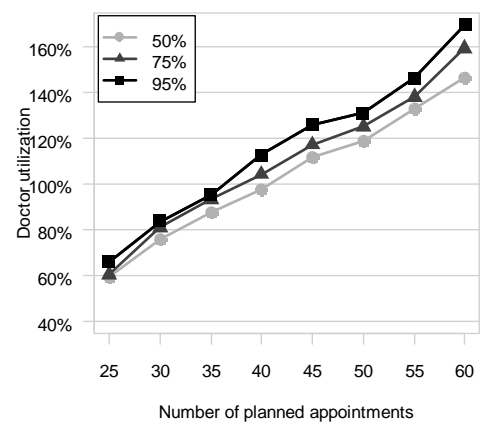

Figure 4: Relationship between performance indicators and number of planned appointments per session in two-doctor consult room. 


\subsection{Results of consult room with three doctors}

Table IV illustrates the results summary of consult room with three doctors. The number of appointment in one session ranges from 25 to 60. Fig. 5 illustrates the relationship between four key performance indicators and the number of planned appointments per session.

It is observed from Fig. 5(a) and Fig. 5(b) that in median case (50 \%), when the number of planned appointments is 40 or less, no queue forms outside the consult room, therefore the patient waiting time is 0 . It is observed from Fig. 5(c) and Fig. 5(d) that in median case (50 $\%)$, doctors do not work overtime when the number of planned appointments is 50 or less.

It is observed from Fig. 5(a) and Fig. 5(b) that in worst case (95 \%), queue forms when the number of planned appointments is 25 and the corresponding waiting time is about 5 minutes. It is observed from Fig. 5(c) and Fig. 5(d) that in worst case (95\%), doctors have to work overtime when the number of planned appointments is 50 or more.

Table IV: Result summary of consult room with three doctors.

\begin{tabular}{|c|c|c|c|c|c|c|c|c|c|c|c|c|}
\hline \multirow[b]{2}{*}{$\mathrm{N}$} & \multicolumn{3}{|c|}{ Queue } & \multicolumn{3}{|c|}{ Waiting time (Mins) } & \multicolumn{3}{|c|}{ Overtime (Mins) } & \multicolumn{3}{|c|}{ Utilization (\%) } \\
\hline & $50 \%$ & $75 \%$ & $95 \%$ & $50 \%$ & $75 \%$ & $95 \%$ & $50 \%$ & $75 \%$ & $95 \%$ & $50 \%$ & $75 \%$ & $95 \%$ \\
\hline 25 & 0 & 0 & 1 & 0 & 0 & 5.1 & 0 & 0 & 0 & 45 & 47 & 50 \\
\hline 30 & 0 & 0 & 2 & 0 & 0 & 9.6 & 0 & 0 & 0 & 56 & 61 & 67 \\
\hline 35 & 0 & 1 & 2 & 0 & 1.2 & 10.3 & 0 & 0 & 0 & 65 & 72 & 74 \\
\hline 40 & 0 & 1 & 2 & 0 & 4.6 & 10.1 & 0 & 0 & 0 & 73 & 79 & 82 \\
\hline 45 & 1 & 2 & 3 & 5.2 & 10.5 & 15.8 & 0 & 0 & 0 & 86 & 88 & 89 \\
\hline 50 & 2 & 3 & 5 & 6.2 & 13.0 & 24.9 & 0 & 7.5 & 10.5 & 90 & 97 & 102 \\
\hline 55 & 5 & 7 & 9 & 22.1 & 29.9 & 40.7 & 26.5 & 29.7 & 31.5 & 107 & 110 & 111 \\
\hline 60 & 5 & 8 & 11 & 23.5 & 39.0 & 53.0 & 32.0 & 42.0 & 55.3 & 111 & 117 & 120 \\
\hline
\end{tabular}

(a) Patient queue vs $\mathrm{N}$

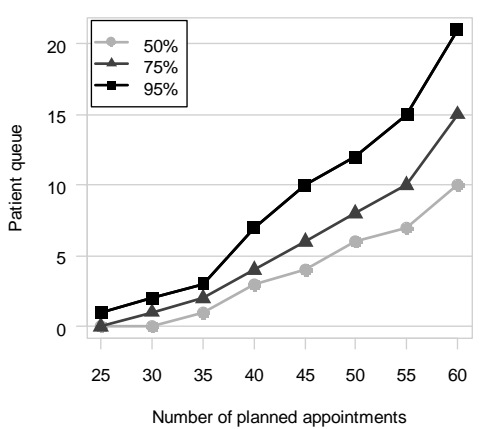

(c) Doctor overtime vs $\mathrm{N}$

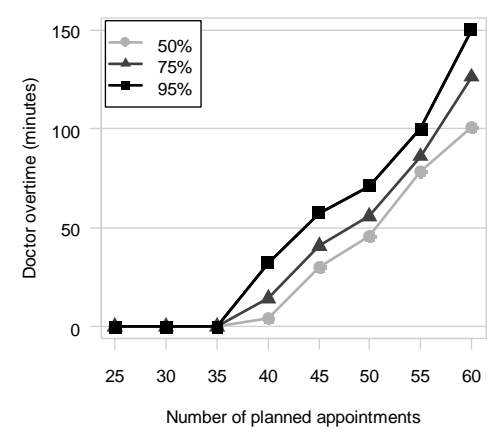

(b) Patient waiting time vs $\mathrm{N}$

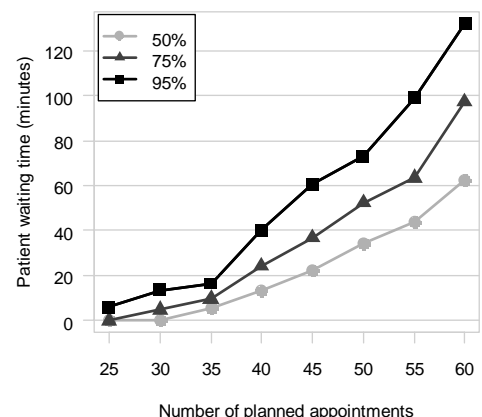

(d) Doctor utilization vs $\mathrm{N}$

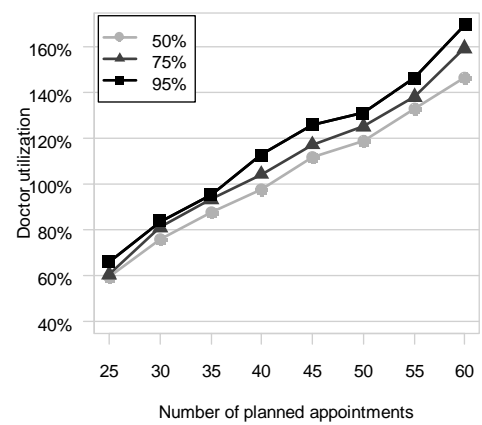

Figure 5: Relationship between performance indicators and number of planned appointments per session in three-doctor consult room. 


\subsection{The optimal number of planned appointments under various circumstances}

In actual practice, one of the challenges SOC schedulers encounter is to determine the optimal number of appointment to be arranged in one session to meet various key performance indicators. Simulation results provide some insights into the relationship between the number of planned appointments and key performance indicators. This aids clinic staff to make more informed and efficient decisions. Some examples are provided below:

If the main requirement is to ensure that no more than 10 patients are waiting outside the consult room at any time, the optimal number of planned appointments per session is 40 for one-doctor consult room, 45 for two-doctor consult room and 55 for three-doctor consult room according to the $95 \%$ simulation results of patient queue.

If the main requirement is to ensure that the median patient waiting time does not exceed 10 minutes, the optimal number of planned appointments per session is 30 for one-doctor consult room, 35 for two-doctor consult room and 50 for three-doctor consult room respectively according to the $50 \%$ simulation results of patient waiting time.

If the main requirement is that the doctors should work overtime in any cases, the optimal number of planned appointments per session is 30 for one-doctor consult room, 35 for twodoctor consult room and 45 for three-doctor consult room respectively according to the $95 \%$ simulation results of doctor overtime.

Finally, if the main requirement is to ensure that the median doctor utilization is about 80 $\%$, the optimal number of planned appointments per session is 30 for one-doctor consult room, 30 for two-doctor consult room and 40 for three-doctor consult room respectively according to the $50 \%$ simulation results of doctor utilization.

\section{CONCLUSION}

This paper analyzed the appointment scheduling systems in specialist outpatient clinics (SOC) to determine the optimal number of appointments to be planned in one session based on different performance indicators and consult room configurations. A discrete simulation model was constructed to describe the daily workflow of SOC. Three types of consult rooms were considered: consult room with one doctor, consult room with two doctors and consult room with three doctors. An appointment rule based on Bailey's rule was proposed to generate appointment schedule. Both the number of appointments at the beginning of a session and the intervals between two successive appointments are flexible according to different consult room configurations.

Extensive ground data were collected to generate model parameters. These include consult time, no-show rate, lateness rate. Schedules with different numbers of planned appointments were tested in the simulation model.

Results of the schedules were measured by key performance indicators including patient queue, patient waiting time, doctor utilization and doctor overtime. Simulation results showed that the optimal number of planned appointments varied according to different performance indicators and consult room configurations. A detailed relationship between the number of planned appointments and four key performance indicators were given in the paper as well.

Simulation results in this paper are based on the several assumptions. For example, all doctors are assumed to be identical. As part of future research effort, difference among doctors can be considered and appointment schedules can be customized to the specific doctor. In this paper, trade-off between key performance indicators is not considered. Such a trade-off can be studied in the future research. Other directions of the future research effort include considering more complex workflow and exploring more efficient appointment rules. 


\section{ACKNOWLEDGEMENTS}

The authors wish to sincerely thank staffs in the Singapore public hospital for providing source data used in this study and professional opinions.

\section{REFERENCES}

[1] http://medical-dictionary.thefreedictionary.com, accessed on 15-12-2008

[2] http://www.wikipedia.org, accessed on 15-12-2008

[3] Browne, R. (2005). Getting it right: walk-in service versus booked and emergency appointments, International Journal of STD \& AIDS, Vol. 16, 88-89

[4] Bailey, N. (1952). A study of queues and appointment systems in hospital outpatient departments, with special reference to waiting-times, Journal of the Royal Statistical Society, Vol. 14, 185-189

[5] Welch, J. D.; Bailey, N. (1952). Appointment systems in hospital outpatient departments, The Lancet, Vol. 259, 1105-1108

[6] Welch, J. D. (1964). Appointment systems in hospital outpatient departments, Operational Research Quarterly, Vol. 15, No. 3, 224-237

[7] Soriano, A. (1966). Comparison of two scheduling systems, Operations Research, Vol. 14, 388397

[8] Liu, L.; Liu, X. (1998). Block appointment systems for outpatient clinics with multiple doctors, Journal of the Operational Research Society, Vol. 49, No. 12, 1254-1259

[9] Vanden Bosch, P. M.; Dietz, C. D.; Sumeoni, J. R. (1999). Scheduling customer arrivals to a stochastic service system, Naval Research Logistics, Vol. 46, 549-559

[10] Lau, H.; Lau, A. H. (2000). A fast procedure for computing the total system cost of an appointment schedule for medical and kindred facilities, IIE Transactions, Vol. 32, No. 9, 833839

[11] Ho, C.; Lau, H. (1992). Minimizing total cost in scheduling outpatient appointments, Management Science, Vol. 38, No. 12, 1750-1764

[12] Wang, P. P. (1993). Static and dynamic scheduling of customer arrivals to a single-server system, Naval Research Logistics, Vol. 40, 345-360

[13] Cayirli, T.; Veral, E. (2003). Outpatient scheduling in health care: a review of literature, Production and Operations Management Society, Vol. 12, No. 4, 519-549

[14] Ho, C.; Lau, H. (1999). Evaluating the impact of operating conditions on the performance of appointment scheduling rules in service systems, European Journal of Operational Research, Vol. 112, 542-553

[15] Hutzschenreuter, A. (2004). Waiting patiently: An analysis of the performance aspects of outpatient scheduling in health care institutes, BMI Paper, Vrije Universiteit, Amsterdam, The Netherlands

[16] Cayirli, T.; Veral, E.; Rosen, H. (2004). Assessment of patient classification in appointment systems, $1^{\text {st }}$ Conference of the POMS College of Service Operations

[17] Cayirli, T.; Veral, E.; Rosen, H. (2006). Designing appointment scheduling systems for ambulatory care services, Health Care Management Science, Vol. 9, 47-58

[18] Su, S.; Shih, C. L. (2003). Managing a mixed-registration-type appointment system in outpatient clinics, International Journal of Medical Informatics, Vol. 70, No. 1, 31-40

[19] Wijewickrama, A. (2006). Simulation analysis for reducing queues in mixed-patients' outpatient department, International Journal of Simulation Modelling, Vol. 5, No. 2, 56-68

[20] http://www.simul8.com/, accessed on 15-12-2008

[21] Simul8 Corporation (2001). Simul8 User Manual, Simul8 Corporation 